\section{Letter to the editor of renewable and sustainable energy reviews}

Re: Comments on "Second law thermodynamic study of heat exchangers: A review", Renewable and Sustainable Energy Reviews 2014; 40: 348-374, by X.T. Cheng, Q. Chen and X.G. Liang

Cheng et al. [1] published deliberately an untruth, which must be corrected. They began their letter with this assertion:

"Heat exchanger effectiveness, $\varepsilon$, does not always increase with decreasing entropy generation or entropy generation number. Especially, both the entropy generation and entropy generation number are zero when $\varepsilon$ is zero. Bejan himself noted this paradox when he analyzed the balanced counterflow heat exchanger and named it "entropy generation paradox" [2]. Bejan explained that the behavior in the $\varepsilon \rightarrow 0$ limit is neither expected nor intuitively obvious because the $\varepsilon \rightarrow 0$ limit will never occur in practical applications. However, this explanation is not satisfactory because it only can explain the $\varepsilon \rightarrow 0$ limit, but cannot remove the paradox."

This is false, in fact, it is the opposite to what I contributed on this topic in the cited book [2]. My contribution was that I showed that there is no paradox. Here is what I wrote in the 1997 edition of my thermodynamics book [2], and I cite :

"Much of the attention devoted to this limit in the literature was centered around the paradoxical conclusion that the irreversibility of such heat exchangers decreases both as $\varepsilon \rightarrow 1$ and as $\varepsilon \rightarrow 0$. I was the first to write about this in 1980 [3], when I published an example used by Professor Myron Tribus in his thermoeconomics class at MIT. Back then I felt that the origin of this paradox was clear and that the 'entropy maximum' (at $\varepsilon=0.5$, Fig. 1) associated with it is of little practical consequence. In the meantime, the subject continued to draw attention in settings (configurations) that are considerably more general than the original balanced counterflow example of Ref. [3]. In fact, the essential idea of the existence of a maximum $N_{S}$ versus $\varepsilon$ was rediscovered independently of Prof. Tribus [4-8]."

Next, I made a thermodynamics teaching opportunity out of the "paradox" brought to my attention by Prof. Tribus. I am the one (not Cheng et al. [1]) who demonstrated that the effectiveness $(\varepsilon)$ is not a relevant measure of the thermodynamic performance of a heat exchanger. I showed that the "paradox" disappears if one considers the effect of heat exchanger size $\left(N_{\text {tu }}\right.$, or $\left.\varepsilon\right)$ not on the heat exchanger alone, but on the whole energy system in which the heat exchanger is an organ. I cite:

"The maximum-entropy paradox [3] constitutes an excellent illustration of the importance of the principle of thermodynamic isolation in the optimization of an engineering component. One physical explanation for the anomalous behavior exhibited in Fig. 1 is that in the $\varepsilon \rightarrow 0$ limit, the stream-to-stream temperature difference $\Delta T$ is practically constant and practically equal to $\left|T_{1}-T_{2}\right|$. In other words, $\Delta T$ is insensitive to the vanishing $N_{t u}$. Consequently, what decreases as $N_{\text {tu }}$ and the heatexchanger area approach zero is the total heat transfer rate between the two streams and along with it, the heat transfer irreversibility. The vanishing $\mathrm{N}_{\mathrm{S}}$ seen in the limit $\varepsilon \rightarrow 0$ is a sign that the heat exchanger disappears as an engineering component. So if $\varepsilon \rightarrow 1$ is the "good" heat-exchanger limit, the $\varepsilon \rightarrow 0$ is the "absent" heat-exchanger limit."

Finally, I wrote an entire section titled "The principle of thermodynamic isolation", which teaches the pitfalls of focusing on the organ instead of the animal. I cite :

Heat exchangers contribute to the overall irreversibility of the installations that incorporate them. This is why the lower left corner of Fig. 1 is technically correct, because an absent heat exchanger $(\varepsilon=0)$ can only contribute zero irreversibility as a heat exchanger $\left(N_{S}=0\right)$.

Consider the greater problem of minimizing the irreversibility of a Brayton-cycle power plant. The power plant has five components, which proceeding clockwise, are: heater $(H)$, expander, regenerator (R), cooler (C), and compressor.

Fig. 2a proves that more regenerator (larger $\varepsilon$ ) is always better from the point of view of improving the entire power system.

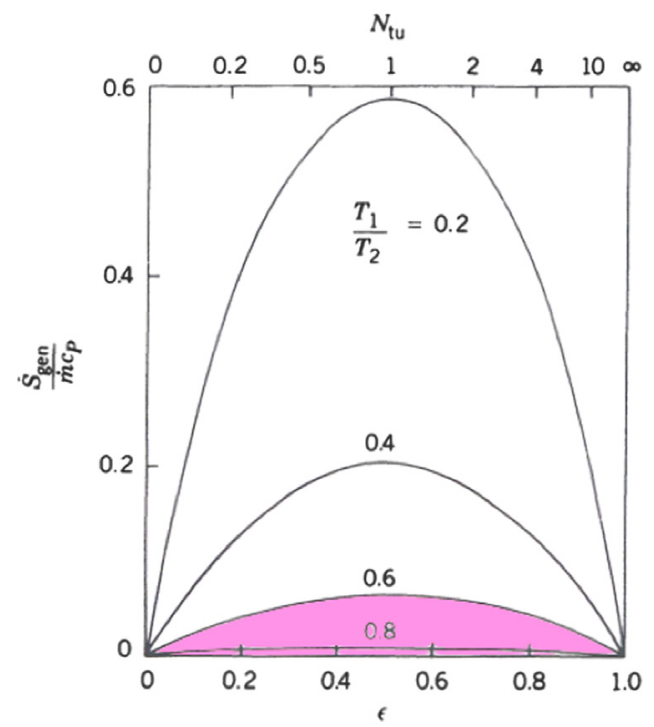

Fig. 1. Entropy generation rate in a balanced counterflow heat exchanger with zero pressure-drop irreversibility $[2,3]$. 
a

b

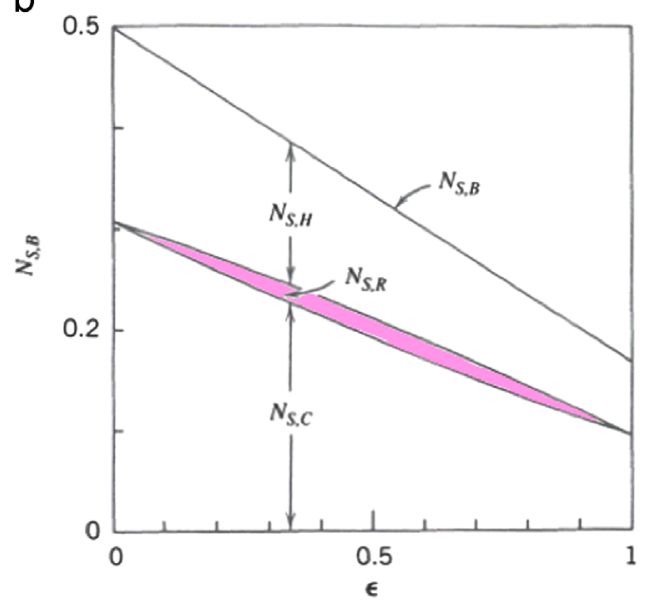

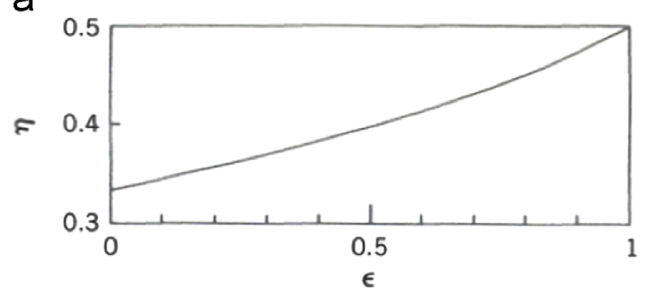

near the base of Fig. 1: zero height at $\varepsilon=0$ and $\varepsilon=1$ and a maximum at $\varepsilon=1 / 2$. The irreversibility of the power plant increase monotonically.

In conclusion, there is no paradox. What remains from Chen et al. assertion is their motive. In an attempt to salvage their own work, they felt it "useful" to denigrate the work of another.

\section{References}

[1] [a] Cheng XT, Chen Q Liang XG. Comments on Second law thermodynamic study of heat exchangers: a review. Renew Sustain Energy Rev 2014;40:348-74;

[b] Cheng XT, Chen Q Liang XG. Comments on Second law thermodynamic study of heat exchangers: a review. Renew Sustain Energy Rev 2015;44:608-10.

[2] Bejan A. Advanced engineering thermodynamics. 2nd ed.. New York: John Wiley \& Sons; 1997.

[3] Bejan A. Second law analysis in heat transfer. Energy 1980;5:721-32.

[4] Sarangi S, Chowdhury K. On the generation of entropy in a counterflow heat exchanger. Cryogenics 1982;22:63-5.

[5] Sekulic DP, Baclic BS. Enthalpy exchange irreversibility. Yugoslavia: University of Novi Sad; 1984. p. 113-23 Faculty of Technical Sciences, No. 15.

[6] da Costa CESM, Saboya FEM, Second law analysis for parallel flow and counterflow heat exchangers, In: Proceedings of the 8th Brazilian Congress of Mechanical Engineers, Sao José dos Campos, S.P., Brazil, pp. 185-187; 1985 Dec.

[7] Sekulic DP. Unequally sized passes in two-pass crossflow heat exchangers: a note on the thermodynamic approach to the analysis. Yugoslavia: University of Novi Sad; 1985\&;1986 Faculty of Technical Sciences, No. 16.

[8] Sekulic DP. Entropy generation in a heat exchanger. Heat Transf Eng 1986;7:83-8.

Fig. 2. (a) Monotonic dependence between Brayton-cycle power plant efficiency and the size of the counterflow heat exchanger (drawn for $r_{\text {large }}=3$ and $r_{\text {small }}=3 / 2$; hence $T_{1} / T_{2}=0.75$ ); (b) distribution of irreversibility among the three heat exchangers: $(H),(R)$, and $(C)[2]$.

Fig. 2b also shows the manner in which $N_{\mathrm{S}, \mathrm{B}}$ is distributed among the three heat exchangers: $\mathrm{H}, \mathrm{R}$, and $\mathrm{C}$.

Sandwiched between $N_{\mathrm{S}, \mathrm{H}}$ and $N_{\mathrm{S}, \mathrm{C}}$ is the irreversibility contribution made by the regenerator; this slice has exactly the same features as the $T_{1} / T_{2}=0.75$ curve that could be drawn
Adrian Bejan

Department of Mechanical Engineering and Materials Science, Duke University, Durham, NC 27708-0300, USA

Received 5 June 2015; accepted 18 September 2015

Available online 10 October 2015 